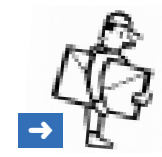

\section{Gedanken zum Begriff der Repräsentativität}

In der Rubrik Forum ZV wurde eine Stellungnahme von Kollege Daniel Schlossberg [1] zum Thesenpapier der FMH Managed Care - Kontrahierungszwang [2] publiziert. Unter Punkt 1 stellt er die Frage: «Was heisst, das FMH-Thesenpapier sei breit abgestützt?» In der Replik hält Jacques de Haller, Präsident der FMH, fest, dass das FMH-Thesenpapier «von einer Gruppe erarbeitet wurde, die für die Ärzteschaft weitgehend repräsentativ ist».

Ich habe mich anlässlich der Publikation dieses Thesenpapiers am 6. September 2006 kurz informiert, welchen Fachgruppen die Mitglieder der Vorbereitungs- und Arbeitsgruppe angehören. Das Resultat meiner kleinen Recherche hat mich überrascht. Da nun Kollege Schlossberg die Frage nach der Repräsentativität dieser Gruppen aufwirft, erlaube ich mir, in Ergänzung zur Antwort unseres FMH-Präsidenten, Jacques de Haller, Herrn Schlossberg noch folgende Informationen nachzureichen: Die Vorbereitungs- und Arbeitsgruppe setzen sich gemäss meinen Recherchen wie folgt zusammen: 10 Allgemeinmediziner, 1 Internist, 1 Gynäkologe, 2 Chirurgen, beide an kantonalen Spitälern tätig, 1 Psychiaterin, der Präsident des Forums Managed Care und der Präsident der VSAO.

Meines Wissens sind in den heute angebotenen Managed-Care-Modellen der freie Zugang zu den Gynäkologen und Psychiatern gewährleistet und wird es sicher auch im Rahmen von kommenden Netzwerken bleiben. Für Gynäkologen und Psychiater wird sich somit durch dieses Thesenpapier wohl nicht viel ändern. Die beiden invasiv an öffentlichen Spitälern tätigen Chirurgen wie auch der Präsident der VSAO repräsentieren situationsgegeben eher nicht die in der Praxis invasiv tätigen Ärzte. Bleiben noch der Präsident des Forums Managed Care, die Allgemeinmediziner und Internisten, welche die Interessen der nicht an Managed-Care-Modellen interessierten Ärzte in dieser Vorbereitungsarbeitsgruppe hätten vertreten können ...

Mitglieder aus 5 Fachgruppen bildeten diese repräsentative Arbeitsgruppe. Im Jahre 2005 verlieh die FMH eidgenössische Weiterbildungstitel an 43 Fachgruppen [3].

\section{Thomas Hofer, Dermatologie FMH, Wettingen}

1 Schlossberg D. Wenn es unter den Fingernägeln juckt. Zum Thesenpapier der FMH Managed Care Kontrahierungszwang. Schweiz Ärztezeitung. 2006; 87(46):1980-1.

2 Thesenpapier FMH. Managed Care - Kontrahierungszwang. Schweiz Ärztezeitung. 2006; 87(36):1536-42.

3 FMH. Ärztestatistik 2005. Schweiz Ärztezeitung. 2006;87(18):747-67.

\section{Replik}

Sehr geehrter Herr Kollege

Es gibt in der Tat Dutzende von unterschiedlichen FMH-Fachtiteln - und noch mehr Ärztegesellschaften. Die Strukturen der FMH haben lange versucht, dieser riesigen Vielfalt Rechnung $\mathrm{zu}$ tragen. In den letzten Jahren jedoch auf enorme Kosten der Effizienz. Deshalb hat der Zentralvorstand vor zwei Jahren eine Strukturreform eingeleitet, die jeder Fachgruppe weiterhin die Möglichkeit bietet, sich Gehör zu verschaffen, aber auch sicherstellt, dass wir gemäss unseren Bedürfnissen agieren und reagieren können. In diesem Sinne tragen die Dachverbände, d.h. die Organisationen, die mehrere Verbände oder Fachgruppen desselben Sektors umfassen, neue und umfangreiche Verantwortlichkeiten.

In der Arbeitsgruppe «Managed Care» waren genau diese Dachverbände vertreten. Selbstverständlich konnten sie frei bestimmen, durch wen sie vertreten werden wollten, ich glaube aber, dass wir nie den Eindruck hatten, die Arbeitsgruppe sei ein abgeschotteter «Club». Bisher hat übrigens auch keine der Dachorganisationen der FMH die bemerkenswerten Ergebnisse der Arbeitsgruppe angezweifelt.

Jacques de Haller, Präsident der FMH 